DOI: https://doi.org/10.24297/jap.v17i.8620

\title{
Black Holes Are Rejuvenating Systems of The Universe
}

\author{
Amrit Šorli Srečko \\ Bijective Physics Institute, Slovenia \\ sorli.bijective.physics@gmail.com
}

\begin{abstract}
Recent research on superfluid quantum vacuum as the physical origin of universal space has opened new perspectives in astronomy and cosmology. Every stellar object is in the active relation with space and its density diminishes according to the mass-energy equivalence principle. As per Newton's Shell Theorem, vacuum density is minimum at the surface of the stellar objects and in their centre. The density of space on the surface of the Black holes and in their centre is so low that atoms become unstable. Therefore, they disintegrate back into the elementary particles and cosmic rays. By transforming old matter into these fresh energies, black holes are rejuvenating the universe and keeping its entropy constant.
\end{abstract}

Keywords: Black Hole Physics, Cosmic Background Radiation, Gravitational Redshift, Cosmology

PACS number: 04.70.- $s, 98.80 .-k$

\section{Introduction}

Geometry plays a very crucial role in Astronomy. The geometry of the cosmos depends upon its density and so for Astronomy, to produce results, one has to start with the shape of the universal space.

NASA's result confirmed by measurements performed by them as well as validated by others have proved, that universal space has Euclidean shape with only a $0.4 \%$ margin of error, and that the space is infinite and "Flat" and the curvature nature of the space in the General Theory of Relativity is only a mathematical description of its actual density. Thus, vacuum density is the origin of the Space [1]. This means that the Euclidean space is the accurate model in cosmology and Riemann's finite geometry cannot be used in the description of the universal space.

Sbitnev's research works suggests that universal space has its physical origin in superfluid quantum vacuum. The $20^{\text {th }}$ century paradigm of "empty" space is replaced with more plausible model, where universal space is not just an abstract mathematical phenomenon, but it has concrete physical properties $[2,3]$.

\section{Mathematical model of vacuum variable density}

Recent research suggests that universal space energy and a given stellar object with mass $m$ are related by massenergy equivalence principle as follows:

$$
\frac{E}{c^{2}}=m=\left(p_{\max }-p_{\min }\right) \cdot V \quad(1) \quad[4]
$$

where $\mathrm{m}$ is the mass of the stellar object, $\rho_{\max }$ is the density of the space in interstellar space, $\rho_{\min }$ is the density of the vacuum on the surface of the stellar object, and $V$ is the volume of the object Space fluctuations that flow from $\rho_{\max }$ towards $\rho_{\min }$ are the origin of gravity, which is the main force of universal dynamics [4]. 
The density of the vacuum $\rho_{\min }$ on the surface of a black hole with the mass of the Sun and radius of 3000 metres is the following:

$$
\begin{aligned}
& \rho_{\text {min }}=\rho_{\text {max }}-\frac{1.989 \cdot 10^{30} \mathrm{~kg}}{1,131 \cdot 10^{11} \mathrm{~m}^{3}} \\
& \rho_{\text {min }}=\rho_{\text {max }}-1.759 \cdot 10^{19} \mathrm{~kg} / \mathrm{m} 3
\end{aligned}
$$

The density of the vacuum $\rho_{\min }$ on the surface of planet Earth is given by the following:

$$
\begin{aligned}
& \rho_{\text {min }}=\rho_{\text {max }}-\frac{5.972 \cdot 10^{24} \mathrm{~kg}}{1,083 \cdot 10^{21} \mathrm{~m}^{3}} \\
& \rho_{\text {min }}=\rho_{\text {max }}-5.514 \cdot 10^{3} \mathrm{~kg} / \mathrm{m}^{3}
\end{aligned}
$$

The density of the vacuum on the surface of a neutron star is $\rho_{\min }=\rho_{\max }-2.0 \cdot 10^{26} \mathrm{~kg} / \mathrm{km}^{3}$ [5] which is $\rho_{\min }=\rho_{\max }-2.0 \cdot 10^{17} \mathrm{~kg} / \mathrm{m}^{3}$

Regarding the maximum density $\rho_{\max }$ which is constant, the density of the vacuum $\rho_{\min }$ on the surface of the black hole is of the order $-10^{19}$. Regarding the maximum density $\rho_{\max }$, the density of the vacuum $\rho_{\min }$ on the surface of the neutron star is of the order $-10^{17}$. Regarding the maximum density $\rho_{\max }$, the density of the vacuum $\rho_{\min }$ on the surface of the planet Earth is of the order $-10^{3}$.

In the interior of the black hole, density of the space $\mathrm{p}$ is increases accordingly to the Newton shell theorem:

$p=p_{\min }+\frac{3 m_{1}}{4 \pi \cdot r^{3}} \quad$ (2) $[4]$,

where $p_{\min }$ is density of the space on the black hole surface, $m_{1}$ is the mass inside the radius $r$ of the shell. Moving deeper inside the black hole, the horizon density of space increases and the atoms there might be stable. When we move close to the centre of the black hole, density of the space decreases and at the centre it is equal as on the surface which is $p_{\text {min }}: r \rightarrow 0, p \rightarrow p_{\text {min }}$.

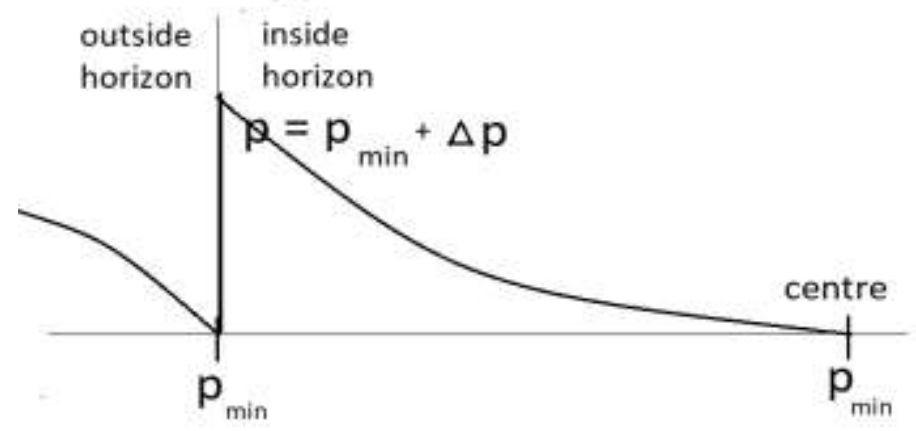

Figure 1: Density of Space Inside Black Hole Horizon 
Black hole is transforming its mass on the horizon and in the centre into elementary particles and further into the energy of space in the form of gravitational waves (thereby eating itself) on the event horizon and at the centre. That's why black holes have tendency to disappear from the visible universe, to contract themselves to the mathematical point.

\section{Density of universal space on the black hole surface, stability of the atom, and matter transformation back into the cosmic rays and gravitational waves}

Considering universal space as the field of energy in which an atom exists, one can ask a reasonable question of relatedness of the atom and variable density of the space. It is logical to predict that for an atom to be stable; it needs a given value of space density. When this value becomes too low, as it is in the case on the surface of the black holes, the atoms become unstable, and falls apart transforming back into the energy of the space in the form of cosmic rays and gravitational waves [4].

This model solves the black holes' puzzle about the disappearance of black holes' matter. I understand that, it is not disappearing rather it is transforming back into the energy of space. Thus "old" matter is transforming back into the "fresh" energy of the space. This model of "Black Holes as the Rejuvenating systems of the Universe" is opening a new prospective in Cosmology.

Back in 1935 Albert Einstein and Nathan Rosen propose the model of wormhole tunes in the universe which connects black holes and white holes. In black hole energy disappears and appears far away in the white hole. The idea proposed in this article is that the white hole actually is universal space itself. Black holes are taking in old matter and transform into fresh energy of space in the form of elementary particles and cosmic rays. Astronomy observation suggests that elementary particles and cosmic rays both have origin in supermassive black holes (SMBH) and neutron stars [6]. I propose that black holes are the rejuvenating systems of the universe. As energy can neither be created nor be destroyed, this article proposes that, the matter in the black holes are continuously disappearing into the space and simultaneously reappearing in the form of elementary particles, cosmic rays and gravitational waves. Thus, the circulation of the energy in the universe runs in a close cycle:

black holes $\rightarrow$ elementary particles + cosmic rays + gravitational waves $\rightarrow$ formation of stellar objects $\rightarrow$ black holes $\rightarrow$ elementary particles + cosmic rays + gravitational waves

This model obeys the first law of thermodynamics and is opening interesting perspectives in cosmology. Thus, problem of energy appearance in the universe is solved. Almost all galaxies have supermassive black holes in their centre [7].

\section{Discussion}

The model presented in this article offers a new interpretation that binary pulsars PSR B1913+16 [8] are losing their orbital velocity, as the mass on their surface is transforming back into the energy of space. Transformation of mass into the energy of space in binary pulsars PSR B1913+16 is causing variable density ripples of space [9], which I suggest in this article are gravitational waves. Thus, Universal space, is not filled with some "hypothetical ether", and is not "empty". Universal space is a type of energy which we call in today's physics as "Superfluid quantum vacuum". I propose in this article that in the centres of binary pulsars PSR B1913+16 mass of the stars in is transformed into elementary particles and further into gravitational waves.

Model presented in this article tends to provide a physical interpretation of event horizon: "Black Hole Scattering: A black hole is formed by the gravitational collapse of a massive object. It is characterized by an event horizon which acts as a one-way membrane in spacetime where matter can enter the horizon, but cannot leave. Several classical gravity and holographic phenomena, such as black hole collisions and quasinormal modes, are described through wave scattering in the black hole spacetime" [10]. Matter can enter the event horizon but cannot leave because it is transformed back into the gravitational waves, elementary particles, and cosmic rays. 
The critical low density of space on the surface of the black holes is a new physical parameter which provides a potential way towards better understanding of the black holes.

The model presented in this article provides a physical interpretation of the interior of the black hole: "The motivation for the present work stems from the need for detailed study of black holes in non-flat backgrounds in comparison and contrast to isolated black holes. A comprehensive investigation of this problem would be a formidable task indeed. In this paper, we have confined ourselves to a specific example that relaxes the condition of asymptotic flatness while preserving time-symmetry. The starting point here is the static black hole in the Einstein universe which belongs to the family of solutions presented by Vaidya. In this spacetime the black hole is well defined as the Killing horizon. However, the nature of the interior of the black hole is not entirely clear. Furthermore, it is not obvious a priori whether the exterior can be matched smoothly to the Schwarzschild vacuum across the black hole surface. We have shown that, this is possible by carrying out this matching using Kruskal coordinates in the two regions. Similarly, we have matched the spacetime to the Einstein universe at the other end. This provides a composite model of a black hole in a non-flat background" [11]. I have shown in this article the physical circumstances of the interior of black hole. At the centre of the black hole there are the same physical circumstances as on the outer side of the horizon.

Galaxy wind comes from the centre of the supermassive black holes $(\mathrm{SMBH})$ which are at the centre of some galaxies. SMBH type of black holes have a mass of 10 million solar masses or more. They are less dense than supernova and their density could be around water density [12]. Because of this relatively low density of SMBH, their axial rotation is shapes them into that of a disk as shown in figure 2.

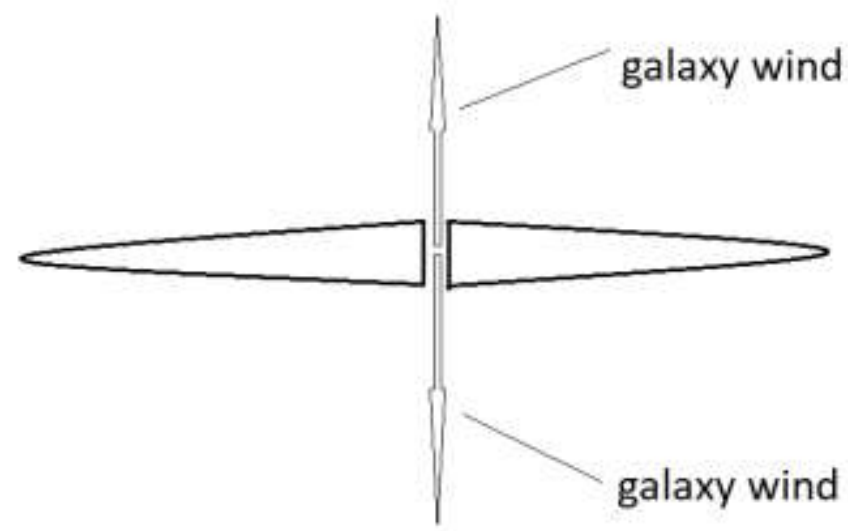

Figure 2: The Disk Shape of SMBH With the Galaxy Wind

In the process of their creation of SMBH a vertical whole is created along the axis of rotation. This happens because of density space $p_{\min }$ (see figure 1 ) at the centre of the SMBH where atoms transform into elementary particles and increases pressure at the centre. The SMBH is "eating" its material from inside around the axis of rotation. The extremely high pressure of the fresh energy created from the mass of the SMBH opens the SMBH in the direction of its axis of rotation and makes the 'Hole'. Energy in the form of elementary particles in the form of cosmic rays comes out from both the sides of the hole with a velocity of 300 to $3000 \mathrm{~km} / \mathrm{s}$ forming galaxy wind. The model of gravity inside event horizon unveils the mystery why supermassive black holes are the most powerful accelerators of elementary particles: "Relativistic magnetized jets from active galaxies are among the most powerful cosmic accelerators, but their particle acceleration mechanisms remain a mystery" [13]. Minimal density of space along the rotation axis of supermassive black hole is the physical circumstance which makes atoms instable. The fall apart and crate high pressure which causes accelerated jets of elementary particles. 
I suggest in this article, the supernova explosion could partially also be due to the minimal density of space $p_{\min }$ at the centre of the star. At the centre of the star, atoms continuously transform into fresh energy in the form of elementary particles leading to increase of pressure till a point where super explosion of the star takes place.

The minimal mass required for the formation of a black star is about 1,4 solar masses. In 1931, Chandrasekhar calculated, that a non-rotating body of electron-degenerate matter above a certain limiting mass at $1,4 M_{\odot}$ has no stable solutions. This today is known as a Chandrasekhar limit [14]. Recent researches confirm that for a star to become a black hole 2,16 solar masses is needed. If the star has less mass than 2,16 solar masses it will develop into a neutron star [15].

In the supernova which is developed from a white dwarf which has the mass below Chandrasekhar limit, the matter is compressed into an earth-sized object with a diameter of between 11000 and $28000 \mathrm{~km}$. The density of a white dwarf is roughly one million time greater than that of the sun, about $1000 \mathrm{~kg} / \mathrm{cm}^{3}$. In white draft centre the transformation of atoms into elementary particles is locked in the very high-density score of the star. When the pressure in the centre has increased to the maximum, that star is exploding into supernova.

In this article is presented the new idea of continuous energy flow in the universe. In black holes atoms continuously falling apart into the elementary particles and cosmic rays which are the "stuff" for the star's formation. Circulation of the energy "black holes - elementary particles - cosmic rays - formation of stars - black holes" is continuous. I suggest that this circulation of the energy in the universe has no beginning and will have no end. In this perspective universe is a non-created system in a permanent dynamic equilibrium. Black holes are transforming "old" matter with high entropy into "fresh" energy in the form of elementary particles and cosmic rays with low entropy. Universe is a system where energy circle is a close loop which maintain the constant level of entropy. Observed increasing entropy of matter in the universe is reversed in black holes. The total entropy of the universe is constant.

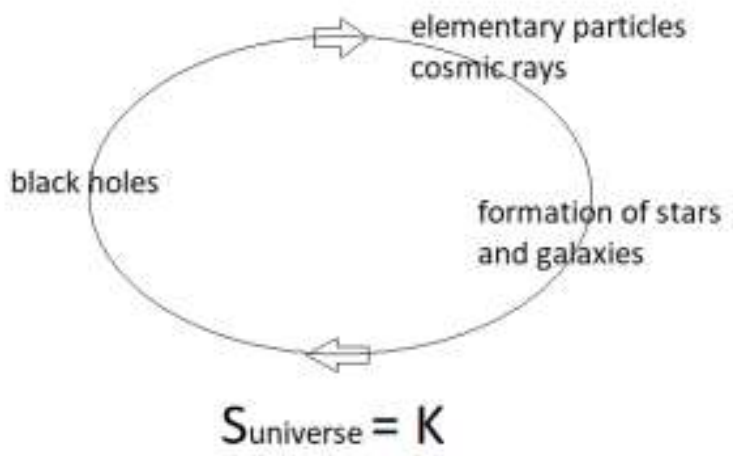

\section{Figure 3: Energy Flow of The Universe Is Permanent, Total Entropy S Is Constant}

According to the first law of thermodynamic energy cannot be created and cannot be destroyed. In the model of the universe presented above, transformation of the energy in the universe is permanent. Not a single Joule of the energy in the universe was ever created. Big Bang model has no answer from where energy of the universe came at the moment of the beginning. Big Bang model suggests that in the inflation phase universe has expanded with the high rate and is still expanding today. NASA has measured universal space is infinite in dimension, it has Euclidean shape [1]. How finite space in the inflation phase has turned into infinite space of today universe Big Bang model also has no answer. CMBR radiation is the radiation measured in existing universal space. Direct reading of data has following conclusion: "Existent universal space is radiating CMBR radiation". There is no prove that this radiation is the relic radiation from the "recombination period". This is just a working hypothesis. And finally, redshift measured by the light coming from distant galaxies can also be read as the "gravitational redshift". Light when polling out of the strong gravity filed of the galaxy is losing some of 
its energy. Gravity vacuum fluctuations are directed from the outer space toward the galaxy and light is moving in the opposite direction [4].

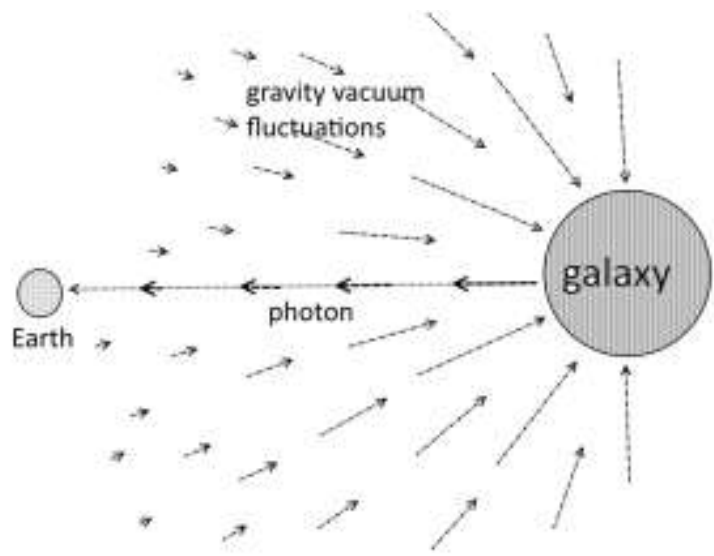

Figure 4: Gravitational Redshift

Swiss astronomer Zwicky has called this effect "tired light" effect [16]. Big Bang model has several weak points which are unsolvable. Universe in permanent dynamic equilibrium where black holes are rejuvenating systems of the universe has no a single weak point and is in accord with all astronomical observations.

Big Bang model predicts that the universe has begun with some hypothetical infinite density, temperature, and pressure. We know from the theory of infinite numbers, that "infinity" is not metrical term. Infinite pressure + 1000 ATM is still infinite pressure. In this sense, the Big Bang theory is not falsifiable and cannot be tested. The model of the universe presented in this article is falsifiable and testable. Astronomical data confirm that black holes are continuously transforming old matter into the fresh energy in the form of elementary particles and cosmic rays. There is no "problem" with the begging, and with the end in the form of "thermal death".

Alan Guth tried to solve the appearance of the energy in the universe by giving gravitational energy negative mathematical sign: "In the inflationary theory the Universe begins incredibly small, perhaps as small as $10^{-24} \mathrm{~cm}$, a hundred billion times smaller than a proton. The expansion takes place while the false vacuum maintains a nearly constant energy density, which means that the total energy increases by the cube of the linear expansion factor, or at least a factor of $10^{75}$. Although this sounds like a blatant violation of energy conservation, it is in fact consistent with physics as we know it.

The resolution to the energy paradox lies in the subtle behavior of gravity. Although it has not been widely appreciated, Newtonian physics unambiguously implies that the energy of a gravitational field is always negative a fact which holds also in general relativity. The Newtonian argument closely parallels the derivation of the energy density of an electrostatic field, except that the answer has the opposite sign because the force law has the opposite sign: two positive masses attract, while two positive charges repel. The possibility that the negative energy of gravity could balance the positive energy for the matter of the Universe was suggested as early as 1932 by Richard Tolman, although a viable mechanism for the energy transfer was not known.

During inflation, while the energy of matter increases by a factor of $10^{75}$ or more, the energy of the gravitational field becomes more and more negative to compensate. The total energy - matter plus gravitational - remains constant and very small, and could even be exactly zero. Conservation of energy places no limit on how much the Universe can inflate, as there is no limit to the amount of negative energy that can be stored in the gravitational field" [17].

I have the following comment: Gravity force is the result of the variable density of the superfluid quantum vacuum which is the physical origin of universal space. Gravity is carried by the quantum structure of universal space [4]. Assigning gravity negative mathematical sign is not allowed because we do not have any experimental 
data gravity has a negative value. Trying to solve with the idea of the negative gravity appearance of energy in the universe does not meet today's standards of research methodology where every element in the model of reality is related to its correspondent model in physical reality with the bijective function of set theory. Universe is set $X$, model of the universe is set $Y$. Every element in the model $Y$ has exactly one corresponded element in the set $\mathrm{X}$, they are related by bijective function:

$$
\begin{array}{r}
f: X \rightarrow Y \\
X:\{1,2,3, \ldots, n\} \\
Y:\{1,2,3, \ldots, n\}
\end{array}
$$

Bijective research methodology excludes existence of negative gravity. Alan Guth's idea we can mathematically write as:

$$
n E_{m}+n\left(-E_{g}\right)=0
$$

At the very beginning, $\mathrm{n}$ is zero and is increasing in inflation. Both of the energies are increasing and their origin still is not explained. Saying that their sum is zero is not solving anything. The model of the universe I propose is built on astronomical data and is without theoretical contradictions. The energy of the universe is non-created, it is just changing the form.

NASA has measured back in 2014 universe space has Euclidean shape [1]. They measured angles between three stellar objects and they have seen the sum of the angles forming the triangle is always 180 degrees. This means Universe has Euclidean shape and is infinite. We will examine Olber's paradox in the perspective of infinity of universal space. To solve Olber's paradox we have to understand first why we have days and we have a night. This is because Earth is rotating around its axis.

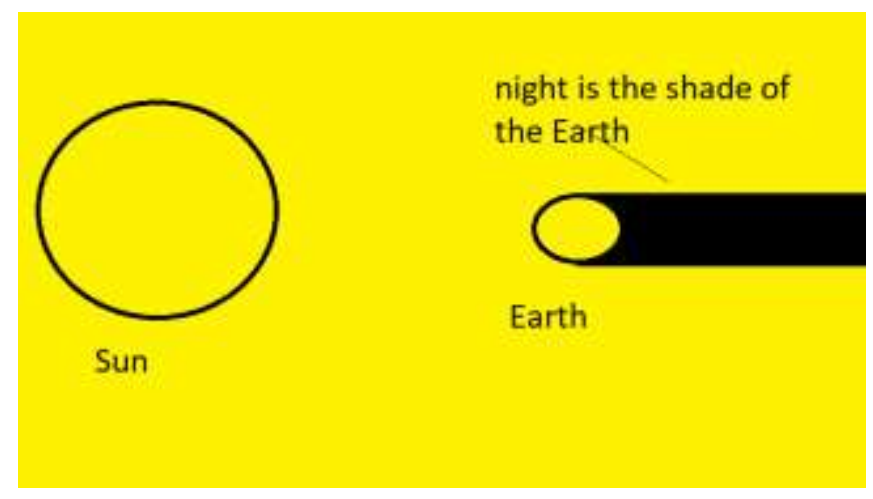

Figure 5: Nigh Is Earth Shade

Earth is rotating, when you are on the Sun side, you have a day. When you are on the other side you have night. Night means you are in the shade of the Earth. We will do a thought experiment: you imagine that Earth stops rotating when you have a day. You will have the only day; the night will be gone. You decide to find another planet and you leave in a fast spaceship. You will need a few months to come far away from the Sun to have night again. Going away from the Sun its luminosity will decrease. After many days of your travel the light will become weaker and weaker, one day you will be in darkness. You will see that the entire universal space is dark. You will see stars as a far distant stellar object with different brightness. The formula for the brightness of the star is following:

$$
b=\frac{L}{4 \pi d^{2}}
$$

where $b$ is the apparent brightness of the star, $L$ is luminosity of the star, and $d$ is the distance to the star. 


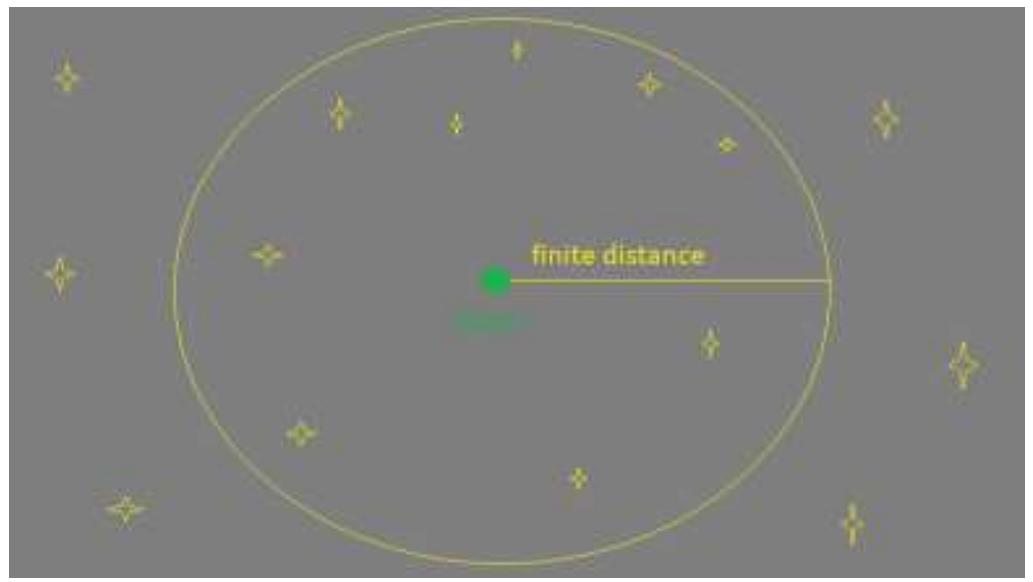

Figure 6: Olber's Paradox Finite Distance Solution

Now we have a solution to Olber's paradox: Earth is rotating around its axis. When we are positioned so that we are away from the Sun, we have a night. Why? Because the brightness of the stars which are in the area of finite distance from us is too low to make us a day when we have a night. The light from the stars which are on infinite distance will never reach us.

The model of the universe as a non-created eternal system is a paradigm shift of cosmology. After finishing the article, I searched what ancient cosmologies say about universe. The only ancient cosmology which clearly supports eternal universe is African Bantu ancient cosmology: "Bantus believe in an eternal, nonchanging universe without a creator" [18]. Term "nonchanging" in ancient Bantus cosmology in the model presented in this article means in the universe time is merely the duration of motion in universal space. Time is not the 4th dimension of space, time is the duration of motion in space when measured by the observer. In the universe, there is no physical past and no physical future [19]. Universal changes run in space which is nonchanging. Buntu ancient cosmology and time as the duration of the change in space are suggesting that the cosmological principle is time-invariant. The general picture of the universe is eternally the same.

\section{Conclusions}

Back holes dynamics is deeply related with the density of space. In the centre of a black hole density of space is equal as on the event horizon. Also, in the centre of the black hole atoms are falling apart into elementary particles and cosmic rays. In white dwarf this transformation creates the high pressure which causes explosion of supernova. In the centre of SMBH atoms are falling apart in elementary particles and cosmic rays which are pervading entire interstellar space. And thus, it can be rightly said: Black Holes are the Rejuvenating systems of the Universe which is a non-created system in a permanent dynamic equilibrium.

\section{References:}

1. NASA https://wmap.gsfc.nasa.gov/universe/uni_shape.html (2014)

2. Sbitnev I.V., Physical vacuum is a special superfluid medium, https://arxiv.org/abs/1501.06763 (2016)

3. Sbitnev I.V. Sbitnev, V.I. Hydrodynamics of the Physical Vacuum: II. Vorticity Dynamics Found Phys (2016) 46: 1238.

4. Amrit Sorli Srecko, Mass-Energy Equivalence Extension onto a Superfluid Quantum Vacuum, Scientific Reports, 9, Article number: 11737 (2019)

5. NASA, https://heasarc.nasa.gov/docs/xte/learning_center/ASM/ns.html (2011). 
6. N.S. Kardashev, Active galactic nuclei and pulsars as cosmic ray sources, Monthly Notices of the Royal Astronomical Society, Volume 326, Issue 3, September 2001, Pages 1122-1126, https://doi.org/10.1046/j.1365-8711.2001.04643.x

7. Kormendy, John; Ho, Luis (2013). "Coevolution (Or Not) of Supermassive Black Holes and Host Galaxies". Annual Review of Astronomy and Astrophysics. 51 (1): 511-653. arXiv:1304.7762

8. Weisberg, J. M.; Nice, D. J.; Taylor, J. H. "Timing Measurements of the Relativistic Binary Pulsar PSR B1913+16". The Astrophysical Journal. 722 (2): 1030-1034. arXiv:1011.0718 (20 October 2010).

9. Taylor, J. H.; Weisberg, J. M. (1982). "A new test of general relativity - Gravitational radiation and the binary pulsar PSR 1913+16". Astrophysical Journal. 253: 908-920.

10. Suraj S. Hegde, Varsha Subramanyan, Barry Bradlyn, and Smitha Vishveshwara, Quasinormal Modes and the Hawking-Unruh Effect in Quantum Hall Systems: Lessons from Black Hole Phenomena, Phys. Rev. Lett. 123, 156802 - Published 11 October 2019

11. K. Rajesh Nayak*, M. A. H. Mac Callum+ and C.V. Vishveshwara, Black Holes in Non-flat Backgrounds: the Schwarzschild Black Hole in the Einstein Universe, https://arxiv.org/pdf/gr-qc/0006040v1.pdf (2000)

12. Celotti, A.; Miller, J.C.; Sciama, D.W. (1999). "Astrophysical evidence for the existence of black holes". Class. Quantum Grav. (Submitted manuscript). 16 (12A): A3-A21. arXiv:astro-ph/9912186

13. Alves E.P. et al., Efficient Nonthermal Particle Acceleration by the Kink Instability in Relativistic JetsPhys. Rev. Lett. 121, 245101, https://arxiv.org/abs/1810.05154 (2018)

14. Venkataraman, G. (1992). Chandrasekhar and his limit. Universities Press. p. 89. ISBN 978-81-7371-035-3.

15. Rezzolla L, Most ER, Weih LR. Using gravitational-wave observations and quasi-universal relations to constrain the maximum mass of neutron stars. The Astrophysical Journal Letters 2018; 852(2): L25.

16. Zwicky F., On the Red Shift of Spectral Lines through Interstellar Space. PNAS 15: 773-779. (1929).

17. Guth. A., "The Beamline" 27, 14, https://ned.ipac.caltech.edu/level5/Guth/Guth3.html (1997).

18. Rene j Herrera and Ralph Garcia-Betrand, DNA, Human Origins, and Migrations, ISBN: 978-0-12-8041246, (2018) https://doi.org/10.1016/C2015-0-01157-1

19. Fiscaletti, D. \& Sorli, A. Perspectives of the numerical order of material changes in timeless approaches in physics. Found. Phys. 45(2), 105-133 (2015). 